\title{
Outcomes following surgery for primary mediastinal nonseminomatous germ cell tumors in the cisplatin era
}

\author{
Kenneth A. Kesler, MD, ${ }^{a}$ Amanda R. Stram, MD, PhD, ${ }^{a}$ Lava R. Timsina, PhD, ${ }^{b}$ Mark W. Turrentine, MD, ${ }^{a}$
} John W. Brown, MD, ${ }^{a}$ and Lawrence H. Einhorn, $\mathrm{MD}^{\mathrm{c}}$

\section{ABSTRACT}

Objective: Treatment of primary mediastinal nonseminomatous germ cell tumors involves cisplatin-based chemotherapy followed by surgery to remove residual disease. We undertook a study to determine short and long-term outcomes.

Methods: A retrospective analysis of patients with primary mediastinal nonseminomatous germ cell tumors who underwent surgery at our institution from 1982 to 2017 was performed.

Results: A total of 255 patients (mean age, 29.2 years) were identified. Acute respiratory distress syndrome occurred postoperatively in 27 patients $(10.9 \%)$, which was responsible for all $11(4.3 \%)$ postoperative deaths. Of patients who developed acute respiratory distress syndrome, more patients received bleomycin-containing chemotherapy (25 out of 169; 14.8\%) than non-bleomycin regimens ( 2 out of 77 ; $2.6 \%)(P=.004)$. With respect to variables independently predictive of longterm survival, evidence of choriocarcinoma before chemotherapy $(n=12)$ was determined to be an adverse factor $(P=.006)$. In contrast, biopsy-proven elements of seminoma $(n=34)$ were predictive of improved survival $(P=.04)$. The worst pathology identified in the residual mediastinal mass after chemotherapy was necrosis in 61 patients $(25.0 \%)$, teratoma in 84 patients (34.4\%), and malignant (persistent germ cell or non-germ cell cancer) in 97 patients (39.8\%), which influenced overall survival $(P<.001)$. Additionally, teratoma with stromal atypia $(n=18)$ demonstrated decreased survival compared with teratoma without atypia $(n=66$; $P=.031)$. Patients with malignancy involving $>50 \%$ of the residual mass $(n=47)$ had a 2.3-fold increased risk of death compared with $\leq 50 \%$ malignancy $(n=45$; $P=.008)$. Finally, elevated postoperative serum tumor markers $(n=40)$ was significantly predictive of adverse survival $(P<.001)$.

Conclusions: In the treatment of primary mediastinal nonseminomatous germ cell tumors, avoiding bleomycin-containing chemotherapy is important. Pre- and postchemotherapy pathology and postoperative serum tumor markers are independent predictors of long-term survival. (J Thorac Cardiovasc Surg 2021;161:1947-59)

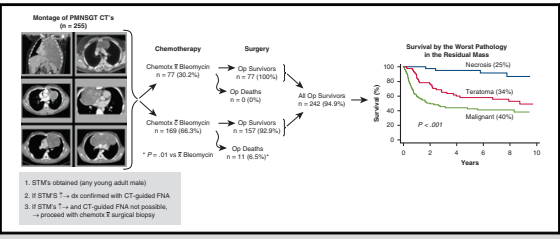

Diagnostic approach, treatments, short- and longterm outcomes of $\mathbf{2 5 5}$ patients with PMNSGCT.

\section{CENTRAL MESSAGE}

Avoiding bleomycin-containing chemotherapy in the treatment of primary mediastinal nonseminomatous germ cell tumors is important. Pre- and postchemotherapy pathology as well as postoperative serum tumor markers are independent predictors of long-term survival.

\section{PERSPECTIVE}

Primary mediastinal nonseminomatous germ cell tumors represent a rare but important malignancy, which occur in otherwise young and healthy patients. Treatment is challenging and involves cisplatin-based chemotherapy followed by surgery to remove residual disease. This study represents the largest single-institutional series in the cisplatin era to define short- and long-term outcomes after surgery.

See Commentaries on pages 1960 and 1961.

\footnotetext{
From the a Division of Cardiothoracic Surgery, Department of Surgery, Indiana University Melvin and Bren Simon Cancer Center, Indianapolis, Ind; ${ }^{\mathrm{b} C e n t e r}$ for Outcomes Research, Department of Surgery, Indiana University, Indianapolis, Ind; and ${ }^{\mathrm{c}}$ Division of Medical Oncology, Department of Medicine, Indiana University Melvin and Bren Simon Cancer Center, Indianapolis, Ind.

Read at the 99th Annual Meeting of The American Association for Thoracic Surgery, Toronto, Ontario, Canada, May 4-7, 2019.

Received for publication May 4, 2019; revisions received Jan 16, 2020; accepted for publication Jan 27, 2020; available ahead of print April 22, 2020.

Address for reprints: Kenneth A. Kesler, MD, Division of Cardiothoracic Surgery, Department of Surgery, Indiana University, 545 Barnhill Dr, EM \#212, Indianapolis, IN 46202 (E-mail: kkesler@iupui.edu).

$0022-5223 / \$ 36.00$

Copyright (c) 2020 by The American Association for Thoracic Surgery https://doi.org/10.1016/j.jtcvs.2020.01.118
}

Although the majority of nonseminomatous germ cell tumors (NSGCTs) originate in the gonads, $5 \%$ to $10 \%$ arise within the anterior mediastinum, which represents the most

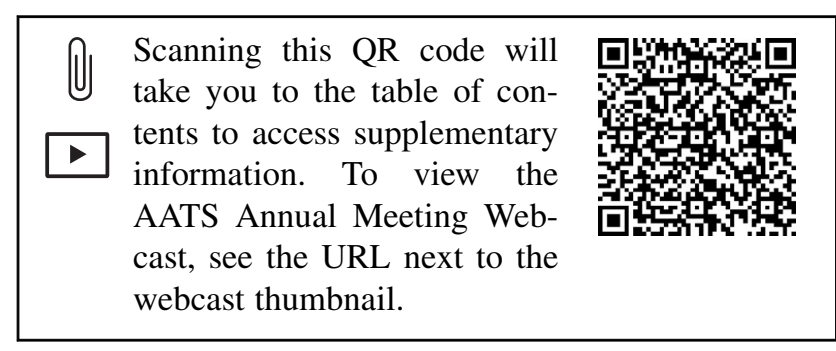




\section{Abbreviations and Acronyms \\ AFP $=$ alpha-fetoprotein \\ ARDS = acute respiratory distress syndrome \\ BHCG = beta subunit of human chorionic gonadotropin \\ CT $=$ computed tomography \\ NSGCT $=$ nonseminomatous germ cell tumor \\ PMNSGCT $=$ primary mediastinal nonseminomatous germ cell tumor \\ STM = serum tumor markers \\ SVC = superior vena cava \\ VIP $=$ etoposide, ifosfamide, cisplatin}

common site of extragonadal origin. The treatment of testicular NSGCTs with cisplatin-based chemotherapy regimens, followed by surgical resection of residual disease is considered among the most successful models for multimodality cancer therapy with $>80 \%$ overall long-term survival. It has been well established that although histologically and serologically identical to their testicular counterparts, primary mediastinal nonseminomatous germ cell tumors (PMNSGCTs) represent a biologically distinct subset of NSGCTs with a $40 \%$ to $50 \%$ overall survival, which places PMNSGCTs in a poor risk category along with other subsets of testicular NSGCTs. ${ }^{1-5}$ Moreover, surgery can be challenging because chemotherapy typically results in fibrosis of mediastinal tissues surrounding residual disease. We undertook a retrospective study representing a 10-year update of our previous institutional report to further define short and long-term outcomes after surgery for PMNSGCTs. ${ }^{6}$

\section{MATERIALS AND METHODS}

\section{Study Design and Patient Selection}

A prospective institutional database was queried to identify patients with PMNSGCTs who underwent surgery following cisplatin-based chemotherapy from 1982 to 2017. A retrospective analysis was undertaken on all identified patients. Data collection consisted of institutional/outside hospital record review and patient/family and physician contact. Nineteen patients were lost to long-term follow-up. The study protocol was approved by the Institutional Review Board of Indiana University and informed consent was waived.

\section{Institutional Treatment Strategy}

Our institutional practice has been to establish a diagnosis of PMNSGCTs by obtaining serum tumor markers (STMs) in young adult male patients presenting with an anterior mediastinal mass. Any elevation in alpha-fetoprotein (AFP) or significant elevation in beta subunit of human chorionic gonadotropin (ßHCG) $>100 \mathrm{U} / \mathrm{L}$ is considered diagnostic. In patients with diagnostic STM elevation, prompt cytologic confirmation can be obtained where computed tomography (CT)-guided biopsy is deemed feasible but surgical biopsy is not performed. Biopsy, either CT-guided or surgical when CT-guided is not considered feasible, is obtained in cases where AFP is normal and BHCG marginally elevated, potentially indicative of seminoma. Although many patients were diagnosed and subsequently received first and occasionally second-line chemotherapy at the discretion of outside facilities, our institutional practice remained consistent over the study interval with surgery to remove a residual mediastinal mass, if deemed operable, after first-line cisplatin-based chemotherapy, regardless of STM status. Rarely, patients were deemed inoperable, including for example, patients with extensive great artery or middle mediastinal involvement. This strategy included patients who presented with metastatic disease which resolved after chemotherapy. An individualized approach was utilized for patients with metastatic disease which did not resolve with first-line chemotherapy. For patients with normal STM after first-line chemotherapy, nonpulmonary and pulmonary metastases were resected when feasible, particularly if deemed to represent teratoma. Extrathoracic metastases were typically removed as a staged procedure before or after mediastinal surgery. Surgery was undertaken for select patients with elevated STMs and limited areas of pulmonary metastases deemed resectable at the time of surgery to remove the residual mediastinal mass. For patients with elevated STMs after first-line chemotherapy and systemic or extensive pulmonary metastases, second-line chemotherapy, more recently in the form of high-dose chemotherapy with peripheral stem cell transplantation, was given before considering surgery. ${ }^{7}$ Patients with elevated STMs after first-line chemotherapy due to an isolated central nervous system metastasis were treated with stereotactic radiation and/or surgery with central nervous system disease control before removal of mediastinal disease. Although the vast majority of patients in this series received 4 cycles of cisplatin-based chemotherapy before surgery, rare patients demonstrated so-called growing teratoma syndrome, defined by a rapidly growing symptomatic mediastinal mass with decreasing STMs before completion of 4 chemotherapy cycles. ${ }^{8}$ In these cases, chemotherapy was discontinued and urgent surgery undertaken.

The details of our technique to remove residual mediastinal disease have been described previously. ${ }^{6,8}$ In brief, an approach was selected to optimize exposure of technically difficult areas anticipated during surgery. Surgical removal involved en bloc dissection of the residual mass and surrounding involved structures. A balanced surgical approach was utilized, sparing critical structures such as phrenic nerves, main pulmonary arteries, great veins, and cardiac chambers where the residual mass abutted but did not grossly invade with frozen section margin control. In cases where phrenic nerves were removed en bloc, prophylactic diaphragm plication was performed on an individual basis (Video 1). With respect to the great veins, reconstruction was done in all cases where en bloc superior vena

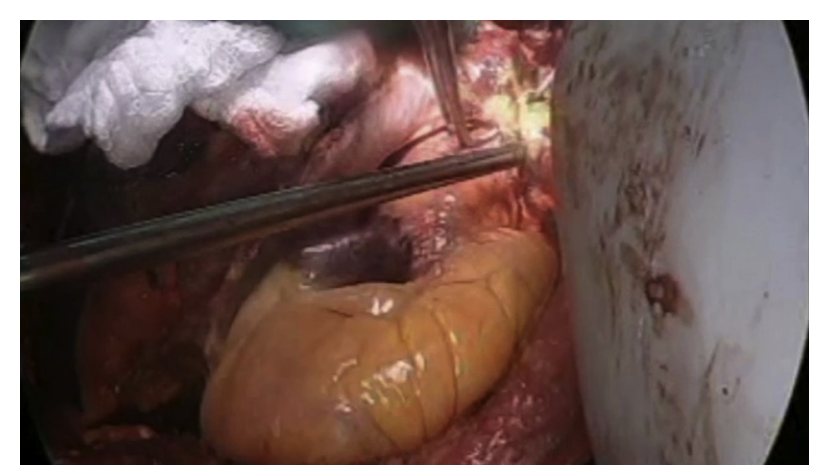

VIDEO 1. We use one of our more challenging PMNSGCT cases to demonstrate the key aspects of our surgical techniques to remove a residual mediastinal mass following cisplatin-based chemotherapy. Video available at: https://www.jtcvs.org/article/S0022-5223(20)30995-8/fulltext. 
cava (SVC) resection was required. If 1 innominate vein was involved, ligation was performed without reconstruction. A single vein reconstruction technique was used for cases where bilateral innominate veins required removal, preferably the right innominate to SVC with ligation of the left innominate vein. Our conduit preference for great vein reconstruction has changed over time. Over the past 6 years of this series, cryopreserved descending thoracic aortic allografts were utilized. Although rarely needed, cardiopulmonary bypass capabilities were made available for select patients. Perioperative fluid and oxygen administration were kept to a minimum, particularly in patients who received bleomycin before surgery.

Patients who presented to surgery with elevated STM had STM measured before hospital discharge and at 1 month postoperatively. Patients with pathologic evidence of viable NSGCTs and normal postoperative STMs were given 2 additional cycles of etoposide/cisplatin. Current practice includes consideration of high-dose chemotherapy for patients with persistently elevated postoperative STMs and recurrent PMNSGCTs.

Routine long-term follow-up included chest radiographs and STM tests every 2 months for the first year, every 4 months for the second year, every 6 months years 3 through 5 , then annually. For patients who pathologically demonstrated a component of teratoma, CT imaging was additionally utilized during follow-up. Patients with recurrent disease were treated on an individual basis, with surgery favored for teratoma and limited areas of malignancy.

\section{Statistical Analysis}

Percentages were calculated using known values of each variable as the denominator. Survival was calculated from the date of surgery. Patients who died within 30 days after surgery or before hospital discharge were considered operative deaths and excluded from long-term survival analysis. Univariate, bivariate, and multivariable analyses were used to examine the primary end points of operative mortality and overall survival. In the univariate analyses, continuous variables were described using mean \pm standard deviation and percentage. In bivariate analyses, $\chi^{2}$ and Fisher exact tests were utilized as deemed appropriate to identify variables potentially predictive of all-cause mortality. The Kaplan-Meier method was used to calculate survival by worst pathology groups (ie, necrosis, teratoma, and malignant). ${ }^{6}$ A secondary survival analyses using the Kaplan-Meier method was also performed comparing outcomes of patients with teratoma with and without pathologic evidence of cellular atypia and patients with $>50 \%$ and $\leq 50 \%$ of the residual mass containing viable cancer. Bivariate Cox proportional-hazard models were used to examine the association between overall survival and each potential variable predicting survival. Multivariable analyses were done using a stepwise estimation method with Cox proportional-hazard model specifying 0.05 as the significant level for removal from the final model and 0.20 for addition to the model. Following are the variables that were used in the multivariable model: STMs at diagnosis, either STM normal or elevated, metastases at diagnosis, prechemotherapy pathology, STM rising at surgery, STM normal at surgery, worst residual mass pathology, age, STM elevated after surgery, and stage. Listwise deletion was used to account for missing data. Finally, due to the small sample size, robust variances were utilized to establish inferences about significance of the Cox model ${ }^{10}$ (Stata/SE version 14.2, StataCorp, College Station, Tex).

\section{RESULTS}

\section{Demographic Characteristics at Presentation}

A total of 255 patients were identified. Average age was $29.2 \pm 8.4$ years. All but 5 patients were men $(98.0 \%)$. Baseline STM status and presence of metastatic disease is given in Table 1 . In summary, $226(95.8 \%)$ patients had elevated STM, AFP, or BHCG, at the time of diagnosis.
TABLE 1. Patient demographic characteristics at time of diagnosis in operative survivors $(N=\mathbf{2 4 4})$

\begin{tabular}{|c|c|}
\hline Demographic characteristic & Result \\
\hline \multicolumn{2}{|l|}{ STMs at diagnosis } \\
\hline \multicolumn{2}{|l|}{$\operatorname{AFP}(n g / m L)(n=236)$} \\
\hline Normal & $21(8.9)$ \\
\hline Elevated & $215(91.1)$ \\
\hline $21-100$ & $14(6.6)$ \\
\hline $101-1000$ & $31(14.6)$ \\
\hline $1001-10,000$ & $93(43.9)$ \\
\hline$\geq 10,000$ & $74(34.9)$ \\
\hline \multicolumn{2}{|l|}{ Missing, $(\mathrm{n}=8)$} \\
\hline \multicolumn{2}{|l|}{ BHCG (IU/L) $(\mathrm{n}=232)$} \\
\hline Normal & $132(56.9)$ \\
\hline Elevated & $100(43.1)$ \\
\hline $2-100$ & $41(41.4)$ \\
\hline $101-1000$ & $35(35.4)$ \\
\hline $1001-10000$ & $13(13.1)$ \\
\hline$\geq 10000$ & $10(10.1)$ \\
\hline \multicolumn{2}{|l|}{ Missing $(\mathrm{n}=12)$} \\
\hline \multicolumn{2}{|l|}{ Either STM $(n=236)$} \\
\hline Both normal & $10(4.2)$ \\
\hline Elevated & $226(95.8)$ \\
\hline \multicolumn{2}{|l|}{ Missing $(\mathrm{n}=8)$} \\
\hline \multicolumn{2}{|l|}{ Metastases at diagnosis } \\
\hline Present $(n=244)$ & $78(32.0)$ \\
\hline Intrathoracic $(\mathrm{n}=244)$ & $58(23.8)$ \\
\hline Lung $(\mathrm{n}=244)$ & $50(20.6)$ \\
\hline \multicolumn{2}{|l|}{ No. } \\
\hline 1 & $12(24.0)$ \\
\hline$\geq 2$ & $38(15.6)$ \\
\hline Mediastinal LNs $(n=244)$ & $13(5.3)$ \\
\hline Extrathoracic $(n=244)$ & $38(15.6)$ \\
\hline LNs $(n=244)$ & $12(4.9)$ \\
\hline Bone $(n=244)$ & $9(3.7)$ \\
\hline Liver/CNS/other $(n=244)$ & $20(8.2)$ \\
\hline
\end{tabular}

Values are presented as $\mathrm{n}(\%)$. STM, Serum tumor marker; $A F P$, alpha fetoprotein $\beta H C G$, beta human chorionic gonadotropin; $L N$, lymph node; $C N S$, central nervous system.

Seventy-eight $(34.1 \%)$ patients presented with evidence of metastatic disease. Two hundred twenty-five patients $(92.2 \%)$ underwent mediastinal biopsy before receiving chemotherapy $(\mathrm{n}=82$, CT-guided and $\mathrm{n}=97$, surgical biopsy). Of the patients who underwent biopsy, $183(81.3 \%)$ demonstrated NSGCT subtypes, including $129(57.3 \%)$ with yolk sac tumors. Forty-two $(18.7 \%)$ and $12(5.3 \%)$ patients had embryonal carcinoma and choriocarcinoma, respectively. Other pathology was identified in 98 $(43.6 \%)$ patients, including teratoma $(\mathrm{n}=52)$, seminoma $(\mathrm{n}=34)$ and malignant transformation into non-germ cell cancer $(\mathrm{n}=12)$.

\section{Chemotherapy and Preoperative STM Status}

All patients received cisplatin-based chemotherapy before surgery. One hundred sixty-nine $(69.7 \%)$ patients 
received bleomycin-containing combination chemotherapy. Twenty-nine of these patients had $\leq 2$ bleomycin cycles, whereas most $(\mathrm{n}=132)$ had $\geq 3$ cycles (mean, $3.5 \pm 0.98$ cycles). Seventy-seven patients received non-bleomycincontaining regimens, the majority of whom $(n=70$; $90.9 \%$ ) received etoposide, ifosfamide, and cisplatin (VIP). One patient received initial high-dose chemotherapy with bone marrow transplant on a clinical trial. Sixteen $(6.6 \%)$ patients received second-line chemotherapy before surgery, including 1 patient who received high-dose chemotherapy with stem cell rescue. Eight $(3.3 \%)$ patients demonstrated a growing teratoma syndrome and underwent surgery before completing 4 cycles of chemotherapy.

TABLE 2. Anatomic structures removed en bloc with the residual mass, and metastatic disease removed after chemotherapy in operative survivors $(N=\mathbf{2 4 4})$

\begin{tabular}{|c|c|}
\hline Variable & Result \\
\hline \multicolumn{2}{|l|}{ Organs removed } \\
\hline Any & $228(93.4)$ \\
\hline 1 & $37(15.2)$ \\
\hline$>1$ & $191(78.3)$ \\
\hline Pericardium & $195(79.9)$ \\
\hline Phrenic nerve & $74(30.3)$ \\
\hline Right phrenic nerve & $19(7.8)$ \\
\hline Left phrenic nerve & $51(20.9)$ \\
\hline Diaphragm plication & $15(6.1)$ \\
\hline \multicolumn{2}{|l|}{ Great vein } \\
\hline Any & $64(26.2)$ \\
\hline Right innominate & $12(4.9)$ \\
\hline Left innominate & $54(22.1)$ \\
\hline Superior vena cava & $25(10.2)$ \\
\hline Inferior vena cava & $1(0.4)$ \\
\hline Cardiac chamber & $9(3.9)$ \\
\hline Right atrium & $5(2.2)$ \\
\hline Left atrium & $2(0.9)$ \\
\hline Left ventricle & $2(0.9)$ \\
\hline Chest wall & $9(3.9)$ \\
\hline Diaphragm & $8(3.5)$ \\
\hline Pulmonary resection & $165(72.3)$ \\
\hline Segment or wedge & $77(33.8)$ \\
\hline Lobectomy & $55(24.1)$ \\
\hline 1 & $35(15.4)$ \\
\hline$>1$ & $20(8.8)$ \\
\hline Pneumonectomy & $12(5.3)$ \\
\hline Postchemotherapy metastases removed & 45 (18.4) \\
\hline Intrathoracic & 34 (13.9) \\
\hline Lung & $28(11.5)$ \\
\hline Mediastinal LNs & $9(3.6)$ \\
\hline Extrathoracic & $15(6.1)$ \\
\hline LNs & $7(2.9)$ \\
\hline Bone & $2(0.08)$ \\
\hline Liver/CNS/other & $6(2.5)$ \\
\hline
\end{tabular}

Values are presented as $\mathrm{n}(\%)$. Left ventricle, Epicardium/superficial myocardium; $L N$, lymph node; $C N S$, central nervous system.
Overall, $149(61.3 \%)$ patients presented to surgery with normal STMs. Of 94 patients with documented elevations in STM at surgery, 84 had elevated AFP and 8 had elevated BHCG. Forty-six patients had rising STMs at the time of surgery. Of note, 21 patients underwent a previous attempt at postchemotherapy resection at outside hospitals before referral.

\section{Surgery}

The operative approaches utilized (in order of frequency) were: median sternotomy $(47.1 \%)$, clamshell with transverse sternotomy $(31.0 \%)$, anterolateral thoracotomy $(17.7 \%)$, and sternotomy combined with separate thoracotomy $(2.9 \%)$. Table 2 lists adjacent structures and suspected metastatic disease removed. The vast majority of patients $(\mathrm{n}=228 ; 93.4 \%)$ had at least 1 adjacent organ removed en bloc with the residual mass. The most common involved structures included pericardium $(\mathrm{n}=195 ; 79.9 \%)$ and lung $(\mathrm{n}=165 ; 72.3 \%)$. Phrenic nerve and great vein resections were required in $30.3 \%$ and $26.2 \%$ of cases, respectively. Cardiopulmonary bypass was utilized in 4 patients who required pneumonectomy due to central pulmonary artery involvement $(\mathrm{n}=2)$ and right atrial free wall resection with patch reconstruction $(n=2)$. Six patients underwent a contralateral pulmonary metastatectomy as a planned staged procedure after recovery from initial surgery. Overall, all but 3 patients had R0 resections, with R1 and $\mathrm{R} 2$ resections in 2 and 1 patients, respectively.

\section{Postoperative Acute Respiratory Distress Syndrome and Mortality}

Twenty-seven (10.9\%) patients manifested acute respiratory distress syndrome (ARDS) postoperatively. Of patients who developed ARDS, significantly more patients had received bleomycin-containing chemotherapy $(\mathrm{n}=25$; $92.6 \%)$ than those who had received chemotherapy regimens without bleomycin $(\mathrm{n}=2 ; 7.4 \%)(P=.004$ [significance adjusted to the extent of pulmonary resection]). There were $11(4.4 \%)$ total postoperative deaths, all of whom died secondary to respiratory failure. Thus, ARDS-related mortality was $40.7 \%$ (11 out of 27 patients). All postoperative deaths occurred in patients who received bleomycin $(n=11 ; 6.5 \%)$, compared with no deaths in patients receiving non-bleomycin regimens $(P=.019)$. With respect to mortality in patients who received bleomycin, there was no significant difference between those receiving $\leq 2$ or $\geq 3$ cycles $(P=.108)$. Of 12 patients who required pneumonectomy, 6 died $(50.0 \%)$, and of these 4 received $>2$ cycles of bleomycin.

\section{Postoperative Pathology and Adjuvant Chemotherapy}

The longest tumor dimension averaged $11.8 \pm 5.3 \mathrm{~cm}$. The worst histology pathologically identified in the residual 
TABLE 3. Worst postchemotherapy pathology in the residual mediastinal mass and metastatic disease removed $(N=244)$

\begin{tabular}{lc}
\hline \multicolumn{1}{c}{ Variable } & Result \\
\hline Postchemotherapy residual mass pathology $(\mathrm{n}=242)$ & \\
Necrosis & $61(25.0)$ \\
Teratoma & $84(34.4)$ \\
Atypia & $18(7.4)$ \\
Nonatypia & $66(27.0)$ \\
Malignant & $97(39.8)$ \\
NSGCT & $76(31.1)$ \\
NGCCA & $16(6.6)$ \\
NSGCT + NGCCA & $5(2.0)$ \\
Type of NGCCA & \\
Adenocarcinoma & $3(1.2)$ \\
Sarcoma & $13(4.1)$ \\
PNET & $5(2.0)$ \\
Unknown & $2(0.8)$ \\
Amount viable* $(\mathrm{n}=97)$ & \\
$\leq 50 \%$ & $45(46.4)$ \\
$>50 \%$ & $47(48.5)$ \\
Unknown & $5(5.1)$ \\
Postchemotherapy metastases pathology $(\mathrm{n}=45)$ & \\
Necrosis & $20(44.4)$ \\
Teratoma & $12(26.7)$ \\
Malignant & $11(24.4)$ \\
NSGCT & $9(81.8)$ \\
Sarcoma & $2(18.2)$ \\
Unknown & $2(4.4)$ \\
\hline
\end{tabular}

Values are presented as $\mathrm{n}(\%) . N S G C T$, Nonseminomatous germ cell cancer; $N G C C A$, malignant transformation into non-germ cell cancer; $P N E T$, primitive neuroectodermal tumor. *The percentage of viable cancer in the residual mass in patients with malignant pathology

mediastinal mass was categorized as complete necrosis $(\mathrm{n}=61 ; 25.0 \%)$, teratoma (with or without stromal atypia) $(\mathrm{n}=84 ; 34.4 \%)$, and malignant (persistent germ cell or malignant transformation into non-germ cell cancer) $(n=97$; $39.8 \%$ ) patients (Table 3 ). With respect to preoperative STM, elevated STM had $47.8 \%$ sensitivity and $70.0 \%$ specificity for pathologic evidence of viable NSGCT in the residual mass.

Thirty-four $(13.9 \%)$ patients underwent removal of mediastinal lymph nodes $(\mathrm{n}=9)$ or residual pulmonary nodules $(\mathrm{n}=28)$. Of these, $14(41.1 \%)$ patients pathologically demonstrated necrosis, $9(26.5 \%)$ demonstrated teratoma, and $4(11.8 \%)$ patients had evidence of malignancy. Fifteen patients underwent removal of extrathoracic metastatic disease, and the worst pathology identified in these specimens was necrosis in $6(40.0 \%)$, teratoma in 2 $(13.3 \%)$, and malignancy in $5(33.3 \%)$. Sixty-three patients received adjuvant cisplatin-based chemotherapy. Five patients underwent pulmonary metastatectomy for recurrent disease. Three of these patients pathologically demonstrated NSGCT, with teratoma and non-germ cell cancer found in the other 2 patients, respectively.

\section{Survival Analysis}

In operative survivors, mean and median follow-up period were 52 and 26 months, respectively, with a range of 1 to 255 months. At the time of last follow-up, 151 patients were alive, and 91 patients had died. Nineteen patients were lost to long-term follow-up. Cancer was the most frequently determined cause of death $(\mathrm{n}=79)$ with 4 patients dying of bone marrow dyscrasias, mainly acute myeloid leukemia. The cause of death was unknown in 7 cases and a cardiac event caused death in 1 patient. Of patients who died as a result of their NSGCT disease, and the site of first recurrence was known, an intrathoracic recurrence was identified in 12 patients, an extrathoracic recurrence in 13 patients, and both intrathoracic and extrathoracic metastases developed in 22 patients.

Multiple variables were analyzed with respect to longterm survival (Table 4). Although larger tumor diameter was associated with higher mortality by univariate analysis, this variable did not remain significant in the multivariate model (Table 5). By univariate analyses, normal STM at time of surgery was protective $(\mathrm{n}=149 ; 61.1 \%)$ $(P=.001)$. Conversely, rising preoperative markers $(\mathrm{n}=46 ; 18.9 \%)$ and elevated AFP $(\mathrm{n}=84 ; 34.4 \%)$ at surgery were predictors of death $(P=.005$ and $P=.001$, respectively), as were elevated postoperative STMs $(\mathrm{n}=40 ; 16.4 \%)(P<.001)$. However, only elevated postoperative STMs remained significantly predictive of adverse survival by multivariate analysis (hazard ratio, $3.41 ; P<.001)$. Patients with biopsy evidence of choriocarcinoma before chemotherapy $(\mathrm{n}=12 ; 5.3 \%)$ had significantly poorer survival by multivariate analysis compared with patients pathologically demonstrating yolk sac tumor or embryonal carcinoma (hazard ratio, 3.07; $P=.006)$. In contrast, biopsy-proven elements of seminoma before chemotherapy was independently predictive of improved survival (hazard ratio, 0.43; $P=.04)$. When eliminating patients who underwent either intrathoracic or extrathoracic metastasectomy with necrosis pathology, our data did not support prognosis based on proposed staging. ${ }^{11}$

The worst pathology category identified in the residual mass following chemotherapy significantly influenced long-term survival, with patients pathologically demonstrating complete tumor necrosis having excellent survival, patients with teratoma having intermediate survival, and patients with malignancy identified having inferior survival $(P<.001)$ (Figure 1 and Figure E1). Additionally, teratoma with stromal atypia $(\mathrm{n}=18)$ demonstrated decreased overall survival compared with teratoma without atypia $(\mathrm{n}=66$; $P=.031$ ) (Figure 2). Finally, patients with malignancy involving $>50 \%$ of the residual mass $(n=47)$ had a 2.3 fold increased risk of death compared with patients with malignancy involving $\leq 50 \%(\mathrm{n}=45 ; P=.008)$ (Figure 3$)$. 
TABLE 4. Proportions of patient survival status and predictors of survival function from univariate Cox regressions

\begin{tabular}{|c|c|c|c|c|c|}
\hline \multirow[b]{2}{*}{ Variable } & \multicolumn{3}{|c|}{ Alive } & \multirow[b]{2}{*}{ Hazard ratio $(95 \%$ confidence interval) } & \multirow[b]{2}{*}{$P$ value } \\
\hline & No & Yes & $P$ value & & \\
\hline \multicolumn{6}{|l|}{ STMs at diagnosis } \\
\hline AFP & & & .97 & & \\
\hline Normal & 8 & 13 & & REF & \\
\hline Elevated & 81 & 134 & & $0.73(0.35-1.51)$ & .389 \\
\hline AFP (ng/mL) & & & .199 & & \\
\hline 21-100 & 3 & 11 & & REF & \\
\hline $101-1000$ & 16 & 15 & & $3.02(0.87-10.43)$ & .081 \\
\hline $1001-10,000$ & 36 & 57 & & $2.11(0.65-6.89)$ & .216 \\
\hline$\geq 10,000$ & 25 & 49 & & $1.73(0.52-5.80)$ & .372 \\
\hline BHCG & & & .097 & & \\
\hline Normal & 44 & 88 & & REF & \\
\hline Elevated & 44 & 56 & & $1.33(0.86-2.06)$ & .199 \\
\hline BHCG (IU/L) & & & .185 & & \\
\hline $2-100$ & 13 & 28 & & REF & \\
\hline $101-1000$ & 17 & 18 & & $1.75(0.83-3.67)$ & .138 \\
\hline $1001-10,000$ & 8 & 6 & & $4.25(1.71-10.56)$ & .002 \\
\hline$\geq 10,000$ & 6 & 4 & & $1.08(0.35-3.35)$ & .892 \\
\hline Either STM & & & 607 & & \\
\hline Both normal & 3 & 7 & & REF & \\
\hline Elevated & 86 & 140 & & $0.976(0.31-3.09)$ & .968 \\
\hline \multicolumn{6}{|l|}{ Metastases at diagnosis } \\
\hline Present & 34 & 44 & .119 & $1.26(0.80-1.98)$ & .325 \\
\hline Intrathoracic & 27 & 31 & .107 & $1.37(0.86-2.18)$ & .192 \\
\hline Lung & 24 & 26 & .088 & $1.38(0.85-2.25)$ & .19 \\
\hline No. & & & .614 & & \\
\hline 1 & 5 & 7 & & REF & \\
\hline$\geq 2$ & 19 & 19 & & $1.32(0.45-3.92)$ & .612 \\
\hline Mediastinal LNs & 8 & 5 & .048 & $1.67(0.77-3.64)$ & .197 \\
\hline Extrathoracic & 17 & 21 & .323 & $1.29(0.74-2.25)$ & .374 \\
\hline LNs & 4 & 8 & $>.999$ & $1.15(0.42-3.14)$ & .783 \\
\hline Bone & 4 & 5 & .732 & $0.98(0.31-3.09)$ & .967 \\
\hline Liver/CNS/other & 11 & 9 & .094 & $1.68(0.87-3.26)$ & .123 \\
\hline \multicolumn{6}{|l|}{ Prechemotherapy pathology } \\
\hline \multicolumn{6}{|l|}{ Nonseminomatous } \\
\hline Yolk sac & 51 & 78 & .79 & $0.90(0.56-1.43)$ & .65 \\
\hline Embryonal & 17 & 25 & .807 & $0.85(0.48-1.50)$ & .569 \\
\hline Choriocarcinoma & 8 & 4 & .064 & $2.12(1.02-4.83)$ & .045 \\
\hline \multicolumn{6}{|l|}{ Other pathology } \\
\hline Seminoma & 8 & 26 & .045 & $0.54(0.26-1.12)$ & .098 \\
\hline Teratoma & 23 & 29 & .356 & $1.03(0.62-1.72)$ & .895 \\
\hline Non-germ cell cancer & 7 & 5 & .145 & $1.70(0.73-3.93)$ & .216 \\
\hline \multicolumn{6}{|c|}{ Postchemotherapy metastases removed } \\
\hline Yes & 22 & 23 & .27 & $1.72(0.81-3.65)$ & .161 \\
\hline Intrathoracic & 18 & 16 & .246 & $1.46(0.63-3.39)$ & .379 \\
\hline Lung & 16 & 12 & .144 & $2.48(0.96-6.35)$ & .06 \\
\hline Mediastinal LNs & 5 & 4 & $>.999$ & $0.44(0.10-1.96)$ & .279 \\
\hline Extrathoracic & 8 & 7 & .389 & $1.20(0.43-3.31)$ & .73 \\
\hline LNs & 3 & 4 & .576 & $1.30(0.12-13.65)$ & .826 \\
\hline Bone & 0 & 2 & .444 & $\mathrm{n} / \mathrm{a}$ & \\
\hline Liver/CNS/other & 5 & 1 & .157 & $1.76(0.49-6.30)$ & .388 \\
\hline Pathology & & & .795 & & \\
\hline Malignant & 6 & 5 & & REF & \\
\hline Teratoma & 16 & 16 & & $0.52(0.21-1.33)$ & .174 \\
\hline
\end{tabular}


TABLE 4. Continued

\begin{tabular}{|c|c|c|c|c|c|}
\hline \multirow[b]{2}{*}{ Variable } & \multicolumn{3}{|c|}{ Alive } & \multirow[b]{2}{*}{ Hazard ratio $(95 \%$ confidence interval $)$} & \multirow[b]{2}{*}{$P$ value } \\
\hline & No & Yes & $P$ value & & \\
\hline AFP at surgery & & & .001 & & \\
\hline Normal & 48 & 110 & & REF & \\
\hline Elevated & 43 & 41 & & $2.02(1.31-3.10)$ & .001 \\
\hline $\operatorname{AFP}(n g / m L)$ & & & .8 & & \\
\hline 21-100 & 17 & 21 & & REF & \\
\hline $101-1000$ & 18 & 15 & & $1.19(0.60-2.37)$ & .628 \\
\hline $1001-10,000$ & 6 & 4 & & $1.88(0.73-4.81)$ & .19 \\
\hline$\geq 10,000$ & 1 & 1 & & $1.12(0.15-8.44)$ & .914 \\
\hline BHCG at surgery & & & .479 & & \\
\hline Normal & 87 & 147 & & REF & \\
\hline Elevated & 4 & 4 & & $1.19(0.38-3.79)$ & .764 \\
\hline BHCG (IU/L) & & & $>.999$ & & \\
\hline $2-100$ & 3 & 3 & & REF & \\
\hline $101-1000$ & 1 & 0 & & - & \\
\hline $1001-10,000$ & - & - & & - & \\
\hline$\geq 10,000$ & 0 & 1 & & - & \\
\hline STM rising at surgery & & & .146 & & \\
\hline No & 68 & 126 & & REF & \\
\hline Yes & 21 & 24 & & $2.04(1.24-3.36)$ & .005 \\
\hline STM normal at surgery & & & .001 & & \\
\hline No & 47 & 46 & & REF & \\
\hline Yes & 44 & 105 & & $0.48(0.31-0.74)$ & .001 \\
\hline Worst RM pathology & & & $<.001$ & & \\
\hline Malignant & 52 & 45 & & $10.76(3.88-29.81)$ & $<.001$ \\
\hline Necrosis & 7 & 54 & & REF & \\
\hline Teratoma & 32 & 52 & & $5.89(2.07-16.72)$ & .001 \\
\hline Atypia & 1 & 7 & & & \\
\hline No atypia & 21 & 45 & & & \\
\hline Amount viable $>50 \% *$ & & & .012 & & \\
\hline No & 17 & 28 & & REF & \\
\hline Yes & 30 & 17 & & $2.31(1.24-4.30)$ & .008 \\
\hline Longest tumor dimension $\dagger$ & 13.3 & 10.9 & .001 & $1.07(1.03-1.12)$ & .001 \\
\hline Age at surgery & 29.0 & 29.3 & .779 & $1.00(0.97-1.02)$ & .899 \\
\hline STM elevated after surgery & & & $<.001$ & & \\
\hline No & 61 & 134 & & REF & \\
\hline Yes & 27 & 13 & & $5.04(3.12-8.14)$ & $<.001$ \\
\hline Stage & & & .23 & & \\
\hline I & 5 & 11 & & REF & \\
\hline II & 66 & 118 & & $1.92(0.70-5.28)$ & .207 \\
\hline IIIA & 18 & 16 & & $2.85(0.96-8.48)$ & .06 \\
\hline IIIB & 2 & 6 & & $1.56(0.28-8.54)$ & .609 \\
\hline
\end{tabular}

Values are presented as n (\%). STM, Serum tumor markers; $A F P$, alpha fetoprotein; $R E F$, reference for comparison; $\beta H C G$, beta human chorionic gonadotropin; $L N$, lymph node; $C N S$, central nervous system; $R M$, residual mediastinal mass after chemotherapy. ${ }^{*}$ If residual mass pathologically contains malignancy, malignancy involves $>50 \%$ of the mass.

$\nmid$ Longest tumor dimension in centimeters.

\section{DISCUSSION}

PMNSGCTs represent a rare but important malignancy. Histologically, these neoplasms are typically mixed, composed of at least 1 NSGCT cancer subtype (eg, yolk sac tumor, embryonal carcinoma, and choriocarcinoma) usually with some form of teratomatous pathology ranging from mature teratoma, to teratoma with immature or atypical elements and occasionally frank malignant transformation of teratoma into so-called non-germ cell cancers. The admixture contains variable amount of these nonseminomatous histologies, as well as malignant seminoma on occasion.

Development of cisplatin-based combination chemotherapy for NSGCTs has been responsible for vastly 
TABLE 5. Multivariate predictors of survival

\begin{tabular}{lcr}
\hline \multicolumn{1}{c}{ Variable } & $\begin{array}{c}\text { Hazard ratio } \\
\mathbf{9 5 \%} \text { confidence interval })\end{array}$ & $\begin{array}{c}\boldsymbol{P} \\
\text { value }\end{array}$ \\
\hline Worst RM Pathology & REF & \\
Necrosis & $7.85(2.56-24.09)$ & $<.001$ \\
Malignant & $4.66(1.52-14.30)$ & .007 \\
Teratoma & & \\
STM after surgery & $\mathrm{REF}$ & \\
Normal & $3.41(1.83-6.33)$ & $<.001$ \\
Elevated & & \\
Prechemotherapy pathology & & \\
Choriocarcinoma & $\mathrm{REF}$ & \\
$\quad \begin{array}{l}\text { No } \\
\text { Yes }\end{array}$ & $3.07(1.37-6.86)$ & \\
Seminoma & & .006 \\
$\quad$ No & $\mathrm{REF}$ & \\
$\quad$ Yes & $0.43(0.19-0.94)$ & \\
\hline
\end{tabular}

$R M$, Residual mediastinal mass after chemotherapy; $R E F$, reference for comparison; $S T M$, serum tumor markers.

improved long-term survival rates compared with outcomes in the pre-cisplatin era. Four courses of bleomycin, etoposide, and cisplatin chemotherapy have traditionally been considered the standard of care for patients with poor-risk NSGCT, including PMNSGCT. The magnitude of postchemotherapy surgery for PMNSGCT is typically high, with potential for pulmonary-related morbidity. Pulmonary toxicity is a well-known consequence of bleomycin. Over the past 15 years, our institution has been using a VIP regimen as the chemotherapy of choice for PMNSGCT, although we continue to operate on patients referred from outside facilities who have received bleomycin-containing regimens. ${ }^{12}$ We previously cited a reduction in postoperative respiratory failure in PMNSGCT patients who received non-bleomycin-containing regimens compared with patients who received bleomycin, despite similar extent of surgery, including pulmonary resections. ${ }^{13}$ In this current update, our institution has gone from a $14.8 \%$ rate of postoperative pulmonary failure, which carried $40.7 \%$ mortality in these otherwise young and healthy patients after bleomycin-containing regimens, to an incidence of $2.6 \%$ in patients who received VIP. Although over this study interval there have been clear improvements in ARDS management, including protective ventilation strategies and extracorporeal membrane oxygenation, which may reduce the mortality of ARDS, ARDS remains a complication with significant associated morbidity. Chemotherapy strategies, which minimize the risks of ARDS, therefore remain important.

Ideally, STMs normalize after chemotherapy, and surgery to remove residual disease is planned in 4 to 6 weeks, allowing bone marrow and functional recovery. The standard of care for testicular NSGCT patients who relapse serologically shortly after first-line chemotherapy involves second-line chemotherapy before considering surgery. Response rates of standard cisplatin-based salvage chemotherapy for PMNSCGTs are notoriously poor, however. ${ }^{14}$ Moreover, whereas elevated STMs are diagnostic of PMNSGCT, these data demonstrate postchemotherapy STMs lacked high sensitivity or specificity for residual NSGCT. Finally, the propensity of PMNSGT to transform into non-germ cell cancers, which are typically STM

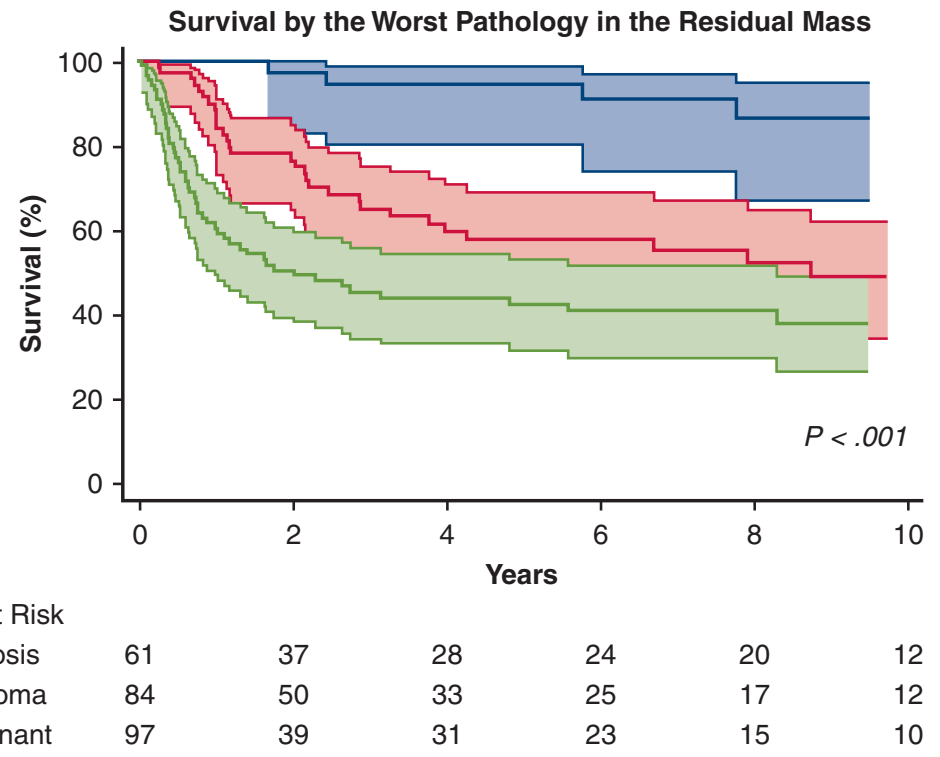

FIGURE 1. Long-term survival in operative survivors with primary mediastinal nonseminomatous germ cell tumors based on the worst pathologic diagnosis microscopically identified in the residual mass (ie, necrosis, teratoma, or malignant). Shaded areas represent respective confidence intervals. Survival was significantly longer in those patients with necrosis, followed by teratoma, and then malignancy $(P<.001)$. The numbers represent patients at risk per given time interval. The $x$-axis timeline starts at 0 years, which refers to 0.08 years, equivalent to 30 days, at the time patient deaths were tracked. 


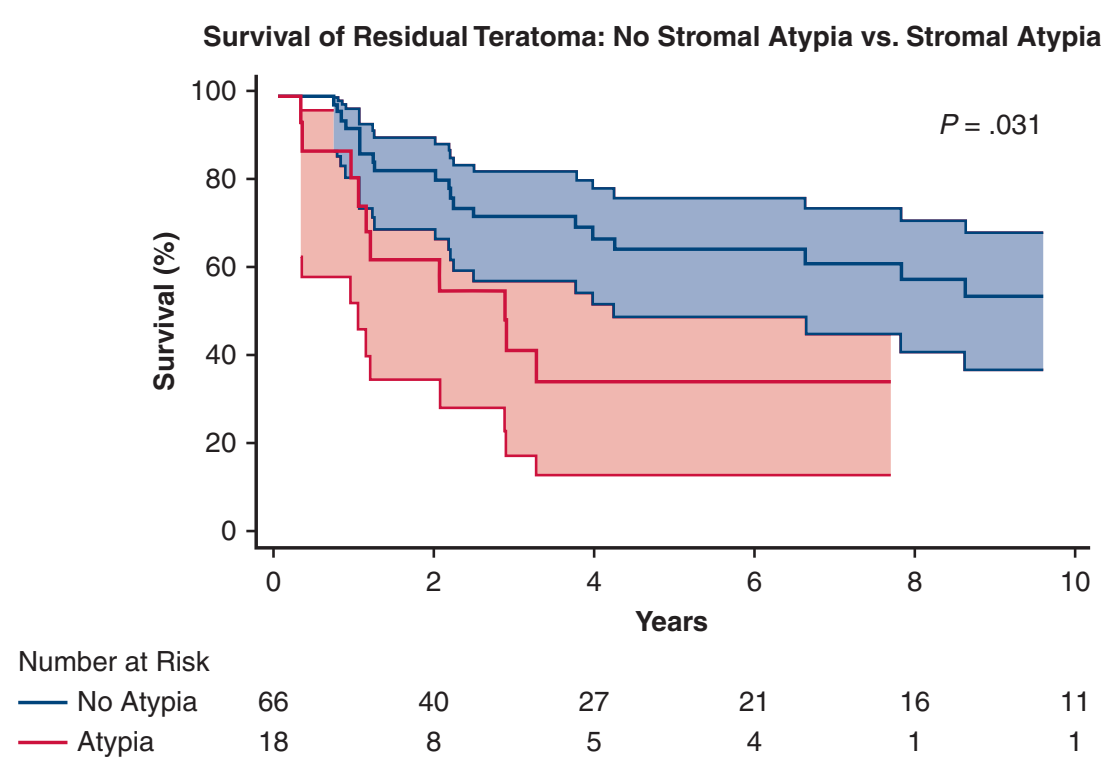

FIGURE 2. Long-term survival in operative survivors with primary mediastinal nonseminomatous germ cell tumors with teratoma as the worst pathology based on the presence or absence of microscopic atypia in the residual mediastinal mass. Shaded areas represent respective confidence intervals. Survival was significantly worse amongst those patients with atypia identified in the residual mass $(P=.031)$. The numbers represent patients at risk per given time interval.

negative as well as refractory to chemotherapy, further questions the role of second-line chemotherapy before surgery. We therefore subscribe to a policy of removing residual disease if deemed operable, regardless of STM status, because the overall results of surgical salvage in patients with residual malignancy after first-line chemotherapy appear to be superior compared with response rates of second-line chemotherapy. ${ }^{15,16}$
STMs appear to remain important from a prognosis standpoint. By univariant analysis, our current study demonstrated that preoperative elevated AFP, elevated STMs in general, and rising STMs were predictive of poor survival, whereas normal STMs was protective. Although elevated postchemotherapy STMs did not remain statistically significant in the multivariate model, persistent elevation of STM after surgery, likely indicative of residual

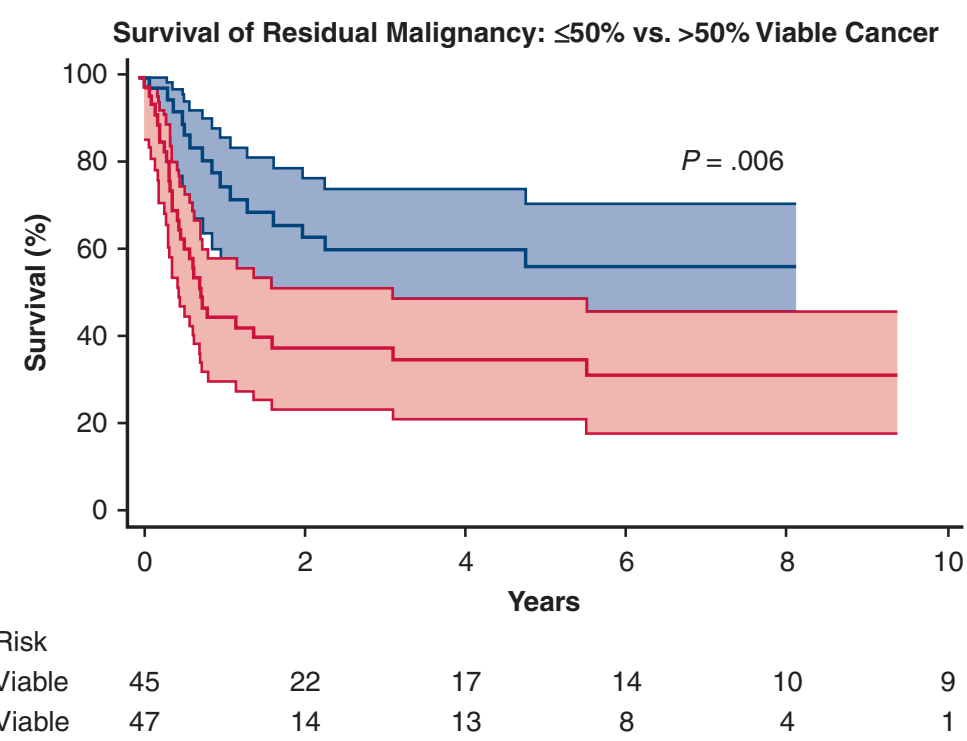

FIGURE 3. Long-term survival of operative survivors with primary mediastinal nonseminomatous germ cell tumors in the subset of patients pathologically demonstrating malignancy (persistent germ cell or transformation into non-germ cell cancer) based on the amount of cancer in the residual mass, $\leq 50 \%$ or $>50 \%$ viable persistent germ cell or non-germ cell cancer. Shaded areas represent respective confidence intervals. Survival was significantly worse amongst those patients with $>50 \%$ viable tumor cells $(P=.006)$. The numbers represent patients at risk per given time interval. 
microscopic NSGCTs, was predictive of adverse survival. Our institutional approach now utilizes high-dose chemotherapy in patients with rising postoperative STMs with an expectation of low but improving cure rates. ${ }^{7}$ A multicenter review of extragonadal NSGCT patients, including 341 with PMNSGCT, identified pretreatment elevated BHCG and nonpulmonary visceral metastases as adverse risk factors. ${ }^{4}$ Of note, less than half of PMNSGCT patients in this study underwent postchemotherapy surgery. Although prechemotherapy tumor histology was not provided, it is plausible that elevated BHCG was a surrogate for the presence of choriocarcinoma, which is independently predictive in our series. Conversely, pure mediastinal seminomas have extremely high cure rates with cisplatin-based chemotherapy alone. ${ }^{3,4}$ Whereas all patients in our series had serologic or pathologic evidence of NSGCTs, it is perhaps not surprisingly the subset of cases with tumors containing a malignant seminoma component had significantly improved survival.

Two staging systems have been proposed for PMNSGCT. A modification of the American Joint Committee on Cancer TNM stage for soft tissue tumors, and a novel staging system described by Moran and Suster. ${ }^{3,11}$ The latter system involves 3 stages ( $I=$ tumor without local invasion or metastases, II = with local invasion and without metastases, and IIIA = intrathoracic metastases/IIIB = extrathoracic metastases), which seems more clinically relevant. There were, however, limitations to this study, including a relatively small patient number and no provision of treatment specifics. A collaborative, multi-institutional study also reported inferior survival in patients presenting with nonpulmonary visceral metastases. ${ }^{3}$ When eliminating patients who had metastatic disease removed pathologically demonstrating tumor necrosis only, the Moran staging system was not predictive in our series. Similarly, intrathoracic or extrathoracic metastatic disease at the time of presentation, or removed after chemotherapy, was not predictive. Unfortunately, attempts to develop a reliable staging system for PMNSGCTs will be confounded by both relatively small patient numbers compared with stages established for other solid tumors and the wide spectrum of benign and malignant pathology in the primary tumor and metastatic disease, when present, following chemotherapy.

Although overall long-term survival averages $40 \%$ to $50 \%$, individual survival after surgery for PMNSGCT has been reported to widely range between $30 \%$ and $90 \%$. Similar to prechemotherapy pathology, pathology identified in resected mediastinal masses can be quite variable and mixed, potentially containing elements of tumor necrosis, teratoma, and malignancy. Current and previous studies from our institution as well as a report from Memorial Sloan Kettering Cancer Center continue to demonstrate the pathology identified in the residual mass following chemotherapy is independently predictive of long-term survival and largely responsible for variable survival rates. ${ }^{6,17} \mathrm{~Pa}$ tients who demonstrate complete tumor necrosis have an excellent long-term prognosis with only a rare late death secondary to recurrent disease (Figure 4). Patients with pathologic evidence of teratoma, with or without tumor necrosis, demonstrate intermediate survival. The poorer prognosis of PMNSGCT compared with testicular NSGCT is not only due to a relative resistance to cisplatin-based chemotherapy but also a higher propensity of teratoma in PMNSGCT to undergo malignant transformation into non-germ cell cancers. ${ }^{18,19}$ Teratoma with stromal atypia arguably represents the precursor to non-germ cell cancer.

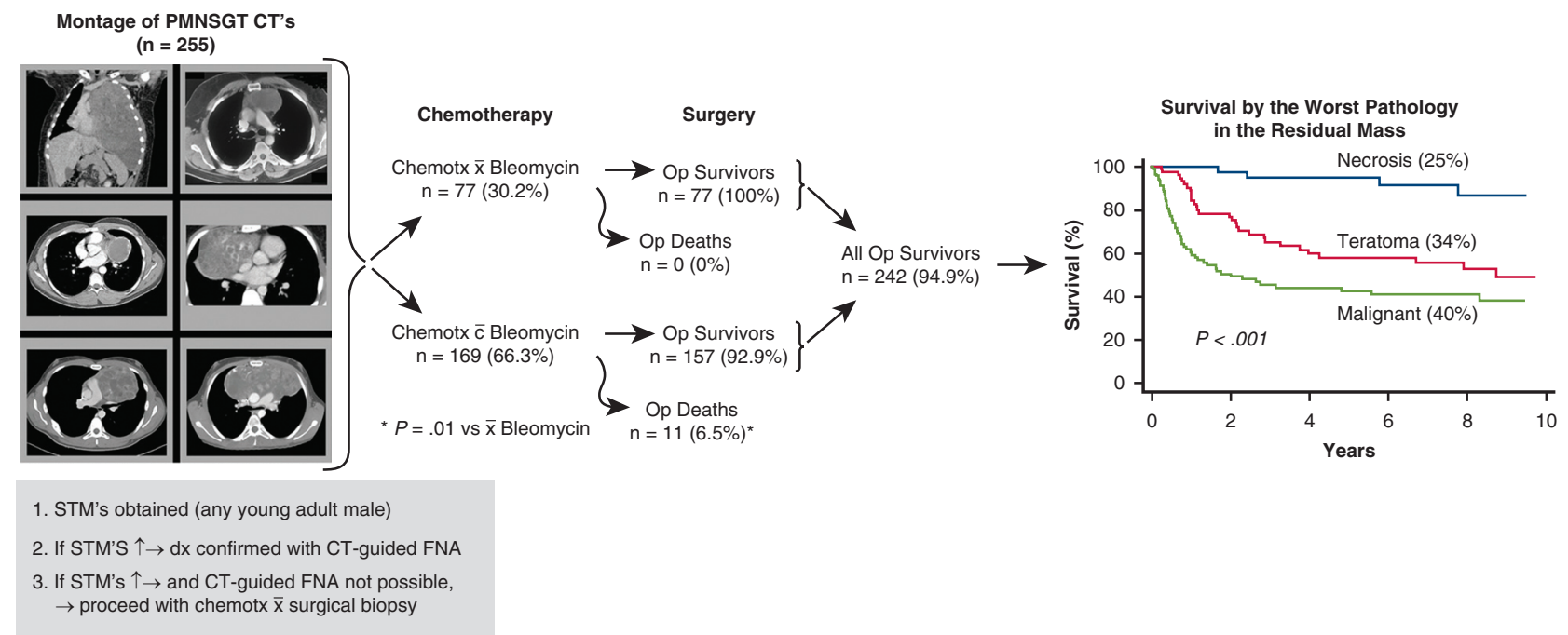

FIGURE 4. Diagnostic approach, treatments, short- and long-term outcomes of 255 patients with primary mediastinal nonseminomatous germ cell tumors (PMNSGCTs). CT, Computed tomography; STM, serum tumor markers; FNA, fine need aspiration; $d x$, diagnosis; chemotx, chemotherapy; $x$, without; $c$, with; $O p$, operative. 
Although sarcomas predominate, the spectrum of nongerm cell pathology in our series is a testimony to the pluripotent nature of these tumors. The subset of patients who pathologically demonstrated teratoma with stromal atypia had long-term survival similar to patients with residual malignancy, which diminished overall survival in the teratoma category in our series. We speculate that pathologic sampling error in large residual masses where small areas of frank non-germ cell cancer are missed, or perhaps observer variability (severe atypia vs frank non-germ cell cancer) could be contributing factors to this finding.

This study further validates the ability of surgery to salvage patients with pathologic evidence of malignancy in the form of either viable NSGCT and/or non-germ cell cancers with poor but possible long-term survival in these cases. The subset of patients with $<50 \%$ of the residual mass containing viable malignancy had long-term survival equivalent to the overall survival of patients whose worst pathology was teratoma. Survival in this current study was significantly diminished when $\geq 50 \%$ of the residual mass contains viable malignancy. This observation was a trend in our 2008 report, which has become statistically significant with additional patients in this updated institutional experience. ${ }^{6}$

Limitations of this study include the single institution and retrospective natures. Accordingly, referral and other biases may exist. Given the outside nature of many referrals, preoperative diagnosis and management strategies were not uniform, and data collection, including long-term followup, was not complete. In this regard, we were unfortunately not able to determine the specific cause of late deaths in all cases. Besides disease-related mortality, other potential causes of late death include myelodysplastic syndromes and cardiovascular disease, to which patients with PMNSGCT appear susceptible. ${ }^{20,21}$ Although mortality due to recurrent disease was most probable in these typically otherwise young and healthy patients, accurate disease-specific survival could not be calculated. An underlying strength of this study is that our institution's case selection, surgical approaches, and perioperative care have remained remarkably constant over the study interval. Finally, it is estimated that $10 \%$ to $20 \%$ of patients with PMNSGCT will be inoperable due to metastatic disease, significant intrathoracic disease, or acute myeloid leukemia. Specific numbers of patients who did not undergo postchemotherapy surgery over this time interval cannot be determined. Inclusion of such cases would clearly negatively influence overall survival.

\section{CONCLUSIONS}

PMNSGCTs represent a challenging group of malignant germ cell tumors. Avoiding bleomycin-containing chemotherapy before these major thoracic surgical procedures is important. Pre- and postchemotherapy pathology, as well as postoperative STMs, are independent predictors of long-term survival. Although overall PMNSGCT survival remains inferior to testicular NSGCTs, an aggressive surgical approach can be justified in these otherwise young and healthy patients.

\section{Webcast}

You can watch a Webcast of this AATS meeting presentation by going to: https://aats.blob.core.windows.net/ media/19\%20AM/Sunday_May5/203BD/203BD/S57\% 20-\%20Mediastinal\%20Tumors/S57_4.mp4.

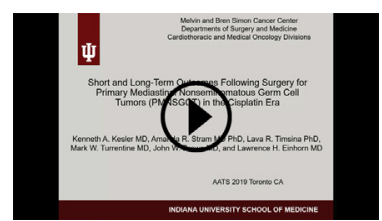

\section{Conflict of Interest Statement}

The authors reported no conflicts of interest.

The Journal policy requires editors and reviewers to disclose conflicts of interest and to decline handling or reviewing manuscripts for which they may have a conflict of interest. The editors and reviewers of this article have no conflicts of interest.

The authors thank Monica Hansome for assisting with data acquisition.

\section{References}

1. Adra N, Einhorn LE. Testicular cancer update. Clin Adv Hematol Oncol. 2017; 15:386-96.

2. Rivera C, Arame A, Jougon J, Velly JF, Begueret H, Dahan M, et al. Prognostic factors in patients with primary mediastinal germ cell tumors, a surgical multicenter retrospective study. Interact Cardiovasc Thorac Surg. 2010;11:585-9.

3. Bokemeyer C, Nichols C, Droz J, Schmoll HJ, Horwich A, Gerl A, et al. Extragonadal germ cell tumors of the mediastinum and retroperitoneum: results from an international analysis. J Clin Oncol. 2002;20:1864-73.

4. Hartmann JT, Nichols CR, Droz JP, Horwich A, Gerl A, Fossa SD, et al. Prognostic variables for response and outcome in patients with extragonadal germcell tumors. Ann Oncol. 2002;13:1017-28.

5. International germ cell consensus classification: a prognostic factor-based staging system for metastatic germ cell cancers. International germ cell cancer collaborative group. J Clin Oncol. 1997;15:594-603.

6. Kesler KA, Rieger KM, Hammoud ZT, Kruter LE, Perkins SM, Turrentine MW, et al. A 25-year single institution experience with surgery for primary mediastinal nonseminomatous germ cell tumors. Ann Thorac Surg. 2008;85:371-8.

7. Adra N, Abonour R, Althouse SR, Albany C, Hanna NH, Einhorn LH. High-dose chemotherapy and autologous peripheral-blood stem-cell transplantation for relapsed metastatic germ cell tumors: the Indiana University experience. J Clin Oncol. 2017;35:1096-102.

8. Kesler KA, Patel JB, Kruter LE, Birdas TJ, Rieger KM, Okereke IC, et al. The "growing teratoma syndrome" in primary mediastinal nonseminomatous germ cell tumors: criteria based on current practice. J Thorac Cardiovasc Surg. 2012;144:438-43.

9. Kesler KA. Technique of mediastinal germ cell tumor resection. Oper Tech Thorac Cardiovasc Surg. 2009; 14:55-65.

10. Lin DY, Wei LJ. The robust inference for the Cox proportional hazards model. $J$ Am Stat Assoc. 1989;84:1074-8.

11. Moran CA, Suster S. Primary germ cell tumors of the mediastinum: III. Yolk sac tumor, embryonal carcinoma, choriocarcinoma and combined nonteratomatous 
germ cell tumors of the mediastinum-a clinicopathologic and immunohistochemical study of 64 cases. Cancer. 1997;80:699-707.

12. Hinton S, Catalano P, Einhorn L, Nichols CR, David Crawford E, Vogelzang N, et al. Cisplatin, etoposide and either bleomycin or ifosfamide in the treatment of disseminated germ cell tumors: final analysis of an intergroup trial. Cancer. 2003:97:1869-75.

13. Ranganath P, Kesler KA, Lawrence LH. Perioperative morbidity and mortality with bleomycin in primary mediastinal nonseminomatous germ cell tumors. $J$ Clin Oncol. 2016;36:4445-6.

14. Hartmann J, Einhorn L, Nichols CR, Droz JP, Horwich A, Gerl A, et al. Secondline chemotherapy in patients with relapsed extragonadal non-seminomatous germ cell tumors: results of an international multicenter analysis. J Clin Oncol. 2001;19:1641-8.

15. Schneider B, Kesler K, Brooks J, Yiannoutsos C, Einhorn LH. Outcome of patients with residual germ cell or non-germ cell malignancy after resection of primary mediastinal nonseminomatous germ cell cancer. J Clin Oncol. 2004;2:1195-200.

16. Radaideh SM, Cook VC, Kesler KA, Einhorn LH. Outcome following resection for patients with primary mediastinal nonseminomatous germ cell tumors and rising serum tumor markers post-chemotherapy. Ann Oncol. 2010;21:804-7.

17. Sarkaria IS, Bains MS, Sood S, Sima CS, Reuter VE, Flores RM, et al. Resection of primary mediastinal non-seminomatous germ cell tumors: a 28 year experience at Memorial Sloan Kettering Cancer Center. J Thorac Oncol. 2011;6:1236-41.

18. Malagon HD, Valdez AM, Moran CA, Suster S. Germ cell tumors with sarcomatous components: a clinicopathologic and immunohistochemical study of 46 cases. Am J Surg Pathol. 2007:31:1356-62.

19. Contreras AL, Punar M, Tamboli P, Tu SM, Pisters L, Moran C, et al. Mediastinal germ cell tumors with an angiosarcomatous component: a report of 12 cases. Hum Pathol. 2010;41:532-7.

20. Mukherjee S, Ibrahimi S, John S, Adnan MM, Scordino T, Khalil MO, et al. Nonseminomatous mediastinal germ cell tumor and acute megakaryoblastic leukemia. Ann Hematol. 2017;96:1435-9.

21. Alanee S, Dynda D. Cardiovascular disease mortality after diagnosis with extragonadal germ cell tumors. J Clin Oncol. 2016;34:1285.

Key Words: mediastinal tumors, germ cell tumors, thoracic surgery, chemotherapy

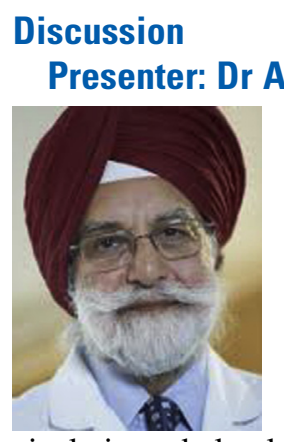

\section{Ananda R. Stram}

Dr Manjit S. Bains (New York, NY). Dr Stram, I enjoyed your paper very much. It is really an excellent review of and update of your vast experience. I don't think anybody else has that extensive an experience. We at Memorial Sloan-Kettering Cancer Center agree that introduction and use of cisplatin and clearly has made a huge difference in the survival of this terrible disease. We also prefer to use bleomycin, etoposide, and platinum-4 cycles of this treatment. Two-thirds of our patients have received that, and the majority show a response in the form of drop in the serum tumor markers. And of course radiologically, you'll see some shrinkage, which is to some degree important, but most important is serum tumor markers. Unlike your experience, if patients at our institution have drop in the serum tumor markers, but they are still high, we would give them second-line treatment before we take them to the operating room for the resection. We find that it is beneficial in those patients. However, if giving them second-line treatment is going to put them in jeopardy for no resectability because the tumor may grow because of poor response, we will go ahead and do the resection.

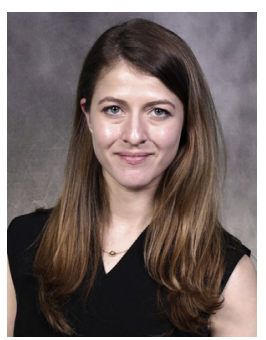

Dr Amanda R. Stram (Indianapolis, Ind). Dr Bains, thank you for your thoughtful and kind comments. We agree, as you allude to, that postchemotherapy pathology in these cases is a spectrum, including teratoma or nongerm cell cancer, both of which are not typically responsive to further chemotherapy, therefore radiographic change cannot be used to assess response to chemotherapy nor criteria for surgery. We also agree that primary mediastinal germ cell patients with persistent or escalating serum markers after chemotherapy represent a very difficult subset. We continue to believe that given both the marginal sensitivity/sensitivity postchemotherapy serum tumor markers have for residual cancer, and the overall poor results of second-line chemotherapy before surgery, that salvage surgery if the residual disease is deemed resectable is preferable in these cases. If surgical resection would likely not be complete, such as in patients with metastatic disease or in rarer cases of patients with significant local disease after first-line chemotherapy, then second-line therapy would be given. At our institution, second-line chemotherapy is typically in the form of high-dose chemotherapy with peripheral stem cell transplant. Certainly, high-dose chemotherapy with peripheral stem cell transplant is a consideration for postoperative patients demonstrating persistent or subsequent serum tumor marker elevation during follow-up with poor but possible long-term survival.

Dr Bains. Also, it's interesting that you make a big point about the use of bleomycin and you have made a point about switching regimen from bleomycin-containing to nonbleomycin-containing etoposide, ifosfamide and cisplatin (VIP). In our experience, early on we found that patients who have had bleomycin, if we maintain low inspired oxygen fraction and not overhydrate them, we have very low incidence of complications. As a matter of fact, in a recent review of our data, about $11 \%$ of patients who had acute respiratory distress syndrome, but we had only 1 death that could be directly attributed to the use of bleomycin. Our oncologists believe that, although there is no difference in the bleomycin-containing versus non-bleomycin-containing regimens as far as response is concerned, patients who are getting the VIP treatment have a higher degree of myelosuppression, nausea, weight loss, thrombocytopenia, anemia, and electrolyte imbalance, as well as dehydration. Patients generally tolerate the VIP worse than the BEP [bleomycin, etoposide, and cisplatin].

Also the VIP, when it is given as the first line, it sort of exhausts a second-line treatment. If a patient has had a VIP treatment, then the second-line treatment may very well be high-dose chemotherapy with stem cell, which we 
still use if the second-line treatment with GIP [gemcitabine, ifosfamide, and cisplatin] fails. I agree that normalization of the serum tumor markers is a great marker for good prognosis, and we try to achieve that as much as we possibly can. Regarding your point about atypia in the teratomas: I'm not sure I have noted that in any of the literature and our pathologists have not addressed that issue, and I guess we will need to do that because of the difference in survival rate that you pointed out, but you may need to elaborate for us as to what your pathologist means by atypia-because when I talk to our pathologists, they say we don't see that much atypia. I'm just wondering, from the question standpoint, whether there is anything special that your anesthesiologist does during the perioperative period and any other thoughts you have that will help us to lower the pulmonary morbidity in these patients.

Dr Stram. Thank you for your institutional perspective. You mentioned all the important intraoperative and postoperative care strategies we also use for our patients with metastatic testicular cancer to the chest and outside referrals of mediastinal germ cell patients who have received preoperative bleomycin. And, we agree that the toxicity of VIP regimens is typically somewhat higher than bleomycin-containing regimens, although this toxicity can usually be mitigated. Surgery for primary mediastinal germ cell cancers is typically much more extensive compared with metastatic germ cell to the chest, as you know. Our data are fairly clear that both the incidence of postoperative acute respiratory distress syndrome and acute respiratory distress syndrome-related mortality is higher after bleomycin-containing regimens, despite equivalent extent of surgery and appropriate intraoperative and postoperative care strategies. In the balance, we believe that avoiding the pulmonary toxicity associated with bleomycin is more important and this strategy would obviously not preclude second-line treatment in form of high-dose chemotherapy with peripheral stem cell transplant.

Teratoma with cellular atypia is an interesting question and in need of further study. We believe there is a spectrum of pathology findings from teratoma without atypia to teratoma with atypia to frankly malignant non-germ cell cancers (often sarcomas). And, there is a spectrum within the category of teratoma with atypia in and of itself. Our pathologists believe that many, and perhaps even most, teratomas in mediastinal germ cell cases demonstrate some degree of atypia.

For the purposes of this study, we only recorded teratoma with atypia when this was stated on the surgical pathology report. Although a number of pathologists were involved with these cases, we believe that teratoma with atypia was used when severely atypical cells were identified. This would include cells that display marked nuclear pleomorphism and marked nuclear irregularities notable even at low magnification. We can only further speculate why survival of patients with pathologic evidence of teratoma containing atypia was more consistent with survival in patients demonstrating malignancy. Potential causes may include sampling error with consequent missing of non-germ cell cancer in these large surgical specimens, or that severe atypia represents a high risk factor for subsequent progression to non-germ cell cancer, or that such atypia is itself incipient non-germ cell cancer before meeting standard microscopic criteria. This may be somewhat analogous to the interobserver variability we see distinguishing Barrett's epithelium with severe dysplasia from adenocarcinoma in situ. Our pathologists are currently reviewing surgical specimens to further define this spectrum.

Dr Bains. Again, compliments with you and your group for excellent results and a great series.

Dr Stram. Thank you again for all your kind and thoughtful comments.

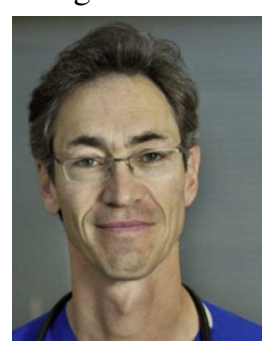

Dr Joseph B. Shrager (Stanford, Calif). Your site also published, I believe, a randomized trial of amiodarone to prevent atrial fibrillation? Do you use amiodarone in people who have received bleomycin?

Dr Stram. Dr Shrager, thank you for this pertinent comment and yes, we have done prospective randomized trials using amiodarone for the prevention of postoperative atrial fibrillation in patients mainly undergoing surgery for lung and esophageal cancer at our institution. Although many of these patients received neoadjuvant therapy, no patient received bleomycin in these randomized studies. Our studies in general demonstrated that amiodarone is effective in reducing the incidence of atrial fibrillation and had no significant adverse pulmonary toxicity compared with controls. That said, the vast majority of the patients in this current mediastinal germ cell study are obviously significantly younger compared with our randomized studies. These young germ cell patients usually present to surgery with a baseline tachycardia following chemotherapy that might worsen after surgery, but the tachycardia is typically not treated and very rarely escalates into a frank atrial tachyarrhythmia. Based our institutional data, we believe there would not be a contraindication to use amiodarone after bleomycin in the rare case it is deemed needed. If bleomycin is avoided, this would obviously also eliminate this potential issue. 


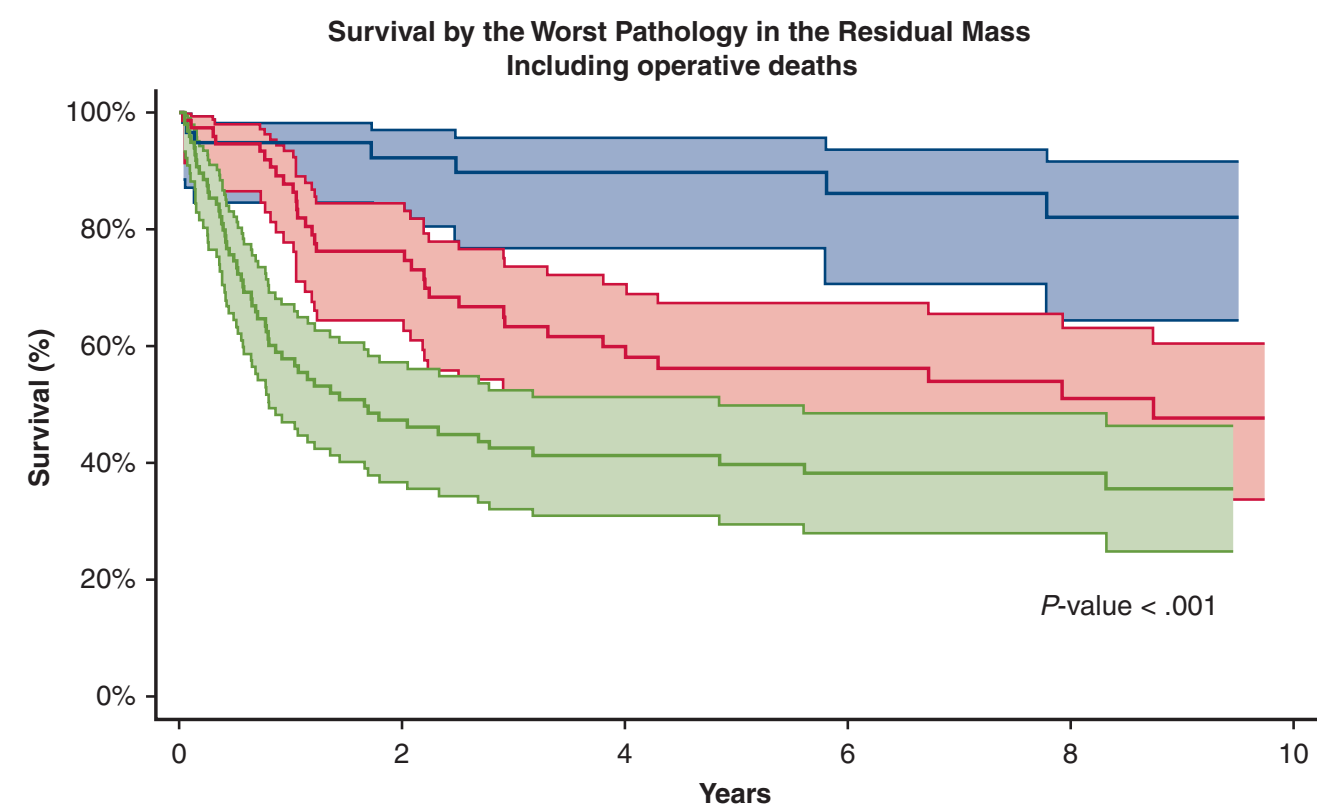

Number at risk

$\begin{array}{cc}\text { Necrosis } & 64 \\ \text { Teratoma } & 86 \\ \text { Malignant } & 103\end{array}$

37

50

39
24

25

23

$\begin{array}{ll}20 & 12 \\ 17 & 12 \\ 15 & 10\end{array}$

FIGURE E1. Long-term survival in patients with primary mediastinal nonseminomatous germ cell tumors, including both operative survivors and operative deaths (within 30 days of surgery), based on the worst pathologic diagnosis microscopically identified in the residual mass (ie, necrosis, teratoma, or malignant). 